\title{
Determination of ganglioside composition and structure in human brain hemangioma by chip-based nanoelectrospray ionization tandem mass spectrometry
}

\author{
Catalin Schiopu - Corina Flangea - Florina Capitan - \\ Alina Serb - Željka Vukelić - Svjetlana Kalanj-Bognar • \\ Eugen Sisu - Michael Przybylski - Alina D. Zamfir
}

\begin{abstract}
We report here on a preliminary investigation of ganglioside composition and structure in human hemangioma, a benign tumor in the frontal cortex (HFC) in comparison to normal frontal cortex (NFC) tissue using for the first time advanced mass spectrometric methods based on fully
\end{abstract}

\section{Schiopu · C. Flangea - A. Serb · A. D. Zamfir ( $₫)$}

Mass Spectrometry Laboratory, National Institute for Research and Development in Electrochemistry and Condensed Matter, Str. Plautius Andronescu Nr. I,

300224 Timisoara, Romania

e-mail: alina.zamfir@uav.ro

C. Flangea $\cdot$ F. Capitan $\cdot$ A. Serb $\cdot$ E. Sisu

"Victor Babes" University of Medicine and Pharmacy,

P-ta Eftimie Murgu Nr. 2,

300041 Timisoara, Romania

\section{Ž. Vukelić $\cdot$ S. Kalanj-Bognar}

Department of Chemistry and Biochemistry,

University of Zagreb Medical School,

Salata 3,

10000 Zagreb, Croatia

E. Sisu

Chemistry Institute of Romanian Academy,

Mihai Viteazul Blvd. 24,

Timisoara 300223, Romania

M. Przybylski

Laboratory of Analytical Chemistry and Biopolymer Structure

Analysis, University of Konstanz,

Universitätsstrabe 10 ,

78464 Konstanz, Germany

\section{A. D. Zamfir}

Department of Chemical and Biological Sciences,

"Aurel Vlaicu" University of Arad,

Revolutiei Blvd 77.

Arad 310130, Romania automated chip-nanoelectrospray (nanoESI) high-capacity ion trap (HCT) and collision-induced dissociation (CID). The high ionization efficiency, sensitivity and reproducibility provided by the chip-nanoESI approach allowed for a reliable MS-based ganglioside comparative assay. Unlike NFC, ganglioside mixture extracted from $\mathrm{HFC}$ was found dominated by species of short glycan chains exhibiting lower overall sialic acid content. In HFC, only GT1 (d18:1/20:0), and GT3 (d18:1/25:1) polysialylated species were detected. Interestingly, none of these trisialylated forms was detected in NFC, suggesting that such components might selectively be associated with HFC. Unlike the case of previously investigated high malignancy gliosarcoma, in HFC one modified $O$-Ac-GD2 and one modified $O$-Ac-GM4 gangliosides were observed. This aspect suggests that these $O$-acetylated structures could be associated with cerebral tumors having reduced malignancy grade. Fragmentation analysis by CID in $\mathrm{MS}^{2}$ mode using as precursors the ions corresponding to GT1 (d18:1/20:0) and GD1 (d18:1/20:0) provided data corroborating for the first time the presence of the common GTla and GT1b isomers and the incidence of unusual GT1c and GT1d glycoforms in brain hemangioma tumor.

Keywords Gangliosides · Brain hemangioma Chip-based nanoelectrospray - Tandem mass spectrometry. Brain tumor biomarker

\section{Introduction}

Hemangioma is a congenital benign tumor or vascular malformation of endothelial cells. Once thought to be strictly congenital, these vascular lesions have been found to occur de novo [1-4]. The disease is characterized by 
grossly dilated blood vessels with a single layer of endotheliurn and an absence of neuronal tissue within the lesions. Cavemous hemangioma, the most common type of brain hemangioma, can be found in any part of the brain as it may occur at any location along the vascular bed [5]. Frontal and temporal lobes are the most common sites of occurrence, with approximately $70 \%$ of these lesions located in the supratentorial region of the brain; the remaining $30 \%$ arise in the infratentorial region [6].

Hemangioma is most commonly diagnosed by routine magnetic resonance imaging (MRI); however, specific detection can be accomplished by gradient-echo sequence MRI that is able to unmask small lesions that may otherwise remain undetected [7]. A practical alternative to these methods is the early detection of hemangioma at an incipient stage, based on routine screening and cancer biomarker discovery before manifestation of clinical symptoms.

Tumorigenesis/malignant transformation is accompanied by aberrant cell surface composition, particularly due to irregularities in glycoconjugate glycosylation pathways. Among molecules bearing characteristic glycosyl epitopes causing such effects are gangliosides, sialic-acid-containing glycosphingolipids (GSLs) incorporated into the outer leaflet of the cell-membrane bilayer [8]. Gangliosides consist of a hydrophobic ceramide (Cer) moiety and a hydrophilic extracellular oligosaccharide chain. Gangliosides contain one or more sialic acids in the form of $\mathrm{N}$ acetylneuraminic acid (Neu5Ac), $N$-glycolylneuraminic acid (Neu5Gc) or derivatives thereof which are attached to the neutral oligosaccharide moiety by an aketosidic linkage.

Ganglioside expression is particularly enriched in mammalian central nervous system (CNS) [9-11] where it is developmentally regulated and closely related to the differentiation state of the cell $[12,13]$. In recent years, ganglioside metabolism, biosynthesis, and physiological functions have been studied extensively. Gangliosides have been implicated in a variety of processes such as cell-cell recognition, cell-substrate interaction, cell adhesion, differentiation, transformation, and intracellular signaling [14-18]. Moreover, several studies showed that brain ganglioside pattern, composition and fine structure are correlated with pathological brain states. In the case of brain tumors, ganglioside expression in the abberant tissue depends on tumor histopathological origin, malignancy grade, and invasiveness $[14,15]$.

In the past, ganglioside composition and quantity in healthy or diseased brain regions and in brain tumor tissues were assessed exclusively by thin layer chromatographic (TLC), immunochemical and immunohistochemical methods [19-21]. However, these techniques have limited effectiveness since they provide information only on major ganglioside species and are unable to detect minor components or ganglioside mixture heterogeneity.
Mass spectrometry is the most precise and sensitive of commonly used bioanalytical methods, able to provide detailed information about the composition of complex ganglioside mixtures and structure of single components with a short analysis time and minimal sample consumption. However, a few studies have demonstrated the potential of nanoelectrospray ionization (nanoESI) and chip-based nanoESI to identify large panels of ganglioside species in normal adult and fetal brains as well as aberrant tumor tissues such as gliosarcoma [22-25].

To elucidate the structure of gangliosides involved in a certain physiological or pathological process we have recently developed novel MS methods based on nanoESI high-capacity ion trap (HCT) multistage $\mathrm{MS}\left(\mathrm{MS}^{\mathrm{n}}\right)$ by collision-induced dissociation (CID) and fully automated chip-based nanoESI HCT CID $\mathrm{MS}^{2}$. We introduced these methods in glycomics of CNS gangliosides and applied them to mapping and structural characterization of ganglioside mixtures isolated from normal and pathological fetal and adult brains [26-28] and for detailed analysis of the oligosaccharide core and ceramide moiety of polysialylated brain ganglioside fractions [29].

In the present study, we optimized our fully automated chip-based nanoESI HCT MS and CID MS ${ }^{2}$ methodology for the first mass spectrometric investigation of ganglioside expression and structure in human brain hemangioma. To identify hemangioma-associated species, a comparative study of ganglioside distribution in the tumoral tissue vs, an ageand gender-matched healthy human brain was performed. To facilitate a rigorous and reliable comparison, the two ganglioside mixtures were extracted, purified, and further analyzed by MS under identical conditions. Comparative screening mass spectra provided direct evidence of differential expression of gangliosides in hemangioma tumor and normal tissue, which allowed postulation of tumor-specific ganglioside structures. Additionally, by CID MS ${ }^{2}$, we were able to collect structural data on the less expressed polysialylated species GT1 (d18:1/20:0) and GDI (d18:1/ $20: 0$ ) that are of potential biomarker value. Obtained results corroborate not only the presence of the common GTla and GTlb isomers but also the incidence of unusual GTlc and GTld glycoforms in hemangioma tumor.

\section{Experimental}

Sampling and characterization of hemangioma and nomal brain tissues

Brain hemangioma localized in the right hemisphere of frontal cortex (HFC) in an adult male patient, age 42 , was clinically diagnosed using computerized tomography (CT) and magnetic resonance imaging (MRI). Brain tumor 
sample was obtained during surgical procedure. The histopathological diagnosis of cavernous hemangioma was confirmed using hematoxylin and eosin stain. This analysis indicated that the tumor tissue was composed of cellular connective tissue and blood spaces with walls varying in thickness, covered with symmetrical endothelium and filled with erythrocytes. The sample of normal frontal cortical brain (NFC) tissue from an age-matched male subject, deceased in a traffic accident, was dissected to serve as control; the brain was obtained from the Department of Forensic Medicine, Faculty of Medicine, Zagreb, Croatia. Tissue samples used for biochemical analysis and ganglioside extraction were weighed and stored at $-20^{\circ} \mathrm{C}$ after careful removal of blood vessels and necrotic elements.

\section{Ganglioside extraction and purification}

Ganglioside extraction was performed using identical procedure and conditions for both tissues. The extraction method followed the protocol developed by Svennerholm and Fredman [30] modified according to Ref. [31]. Each tissue sample was weighed and homogenized in ice-cold distilled water to obtain $10 \%$ homogenate. Lipids were extracted twice using solvent mixture of chloroform: methanol $(1: 2$, by vol.), followed by partition and repartition by adding chloroform, methanol, and water to a final volume ratio 1:1:0.8. The combined upper phases, containing gangliosides were collected. The raw ganglioside extract was purified in several steps: precipitation of coextracted protein-salt complexes followed by centrifugation; low-molecular-weight contaminants were removed by gel-filtration on Sephadex G-25 column and dialysis against water (overnight at $4{ }^{\circ} \mathrm{C}$ ). Finally, the pure extract was evaporated to complete desiccation and weighed.

\section{Sample preparation for MS}

For chip-nanoESI MS analysis, the stock solution of each native ganglioside extract (approximately $0.5 \mathrm{mg} / \mathrm{mL}$ ) was prepared by dissolving the dried material in pure methanol stored at $-27{ }^{\circ} \mathrm{C}$. Working aliquots at concentration of approximately $0.5 \mathrm{pmol} / \mu \mathrm{L}$ (calculated for an average molecular weight of 2,000 ) were obtained by dilution of the stock solution in pure methanol. Methanol was obtained from Merck (Darmstadt, Germany) and used without further purification.

\section{Mass spectrometry}

Mass spectrometry was conducted on a High-Capacity Ion Trap Ultra (HCT Ultra, PTM discovery) mass spectrometer from Bruker Daltonics, Bremen, Germany. All mass spectra were acquired in the mass range $100-3,000 \mathrm{~m} / \mathrm{z}$, with a scan speed of $8,000 \mathrm{~m} / \mathrm{z}$ per second.
$\mathrm{MS}^{2}$ was carried out by CID using He as the collision gas. For tandem MS sequencing, the precursor ions were selected within an isolation width of $2 \mathrm{u}$. Fragmentation spectra were obtained by accumulating scans at variable $\mathrm{rf}$ signal amplitudes within $0.6-1.0 \mathrm{~V}$.

The $m / z$ scale of the mass spectrum was calibrated by use of an external calibration standard G2421 A electrospray "tuning mix," from Agilent Technologies (Santa Rosa, CA, USA). The reference provided in negative ion mode a spectrum with a fair ionic coverage of the $m / z$ range scanned in MS. Under the used experimental conditions, the resolution was below 0.6 Th FWHM across mass range and the obtained mass accuracy was situated within 10 and $80 \mathrm{ppm}$, which represents a normal range of an HCT MS instrument.

The assignment of molecular ions to a certain composition was made by exact mass calculation, while the postulation of oligosaccharide core structures and linkages was based on the previously acquired information [29] and ganglioside biosynthesis pathway principles. Ion designation followed the generally accepted nomenclature introduced by Domon, Costello [32] and revised by Costello et al. [33].

\section{Automated chip-based nanoelectrospray}

Fully automated chip-based nanoelectrospray was performed on a NanoMate robot incorporating ESI 400 Chip technology (Advion BioSciences, Ithaca, USA) controlled and manipulated by ChipSof 8.1 .0 software operating under Windows system. The robot was coupled to the HCT Ultra mass spectrometer by a custom-made mounting system, which allows robot $\mathrm{O}-\mathrm{xyz}$ positioning with respect to HCT counter electrode as described previously [26]. The position of the electrospray chip was adjusted with respect to the sampling cone to give rise to an optimal transfer of the ionic species into the mass spectrometer.

Five-microliter aliquots of each working sample solution were simultaneously loaded into two different wells of the NanoMate 96-microtiter plate. The robot was programmed to aspirate the whole volume of sample, followed by $2 \mu \mathrm{L}$ of air into the pipette tip and afterwards deliver the sample to the inlet side of the 400 microchip. The NanoMate HCT MS system was tuned for operating in the negative ion mode previously demonstrated $[26,27,29]$ as the most appropriate ionization mode for gangliosides. Electrospray was initiated by applying a voltage of $-0.85 \mathrm{kV}$ on the pipette tip, and 0.60 p.s.i. nitrogen back pressure. In order to prevent possible in-source fragmentation, HCT capillary exit was set to $-30 \mathrm{~V}$. The source block was maintained at the constant temperature of $200{ }^{\circ} \mathrm{C}$. Heating of the source block provided an optimal desolvation of the generated droplets without the need of desolvation gas. To prevent 
any cross-contamination or carry-over, following each infusion and MS analysis, the pipette tip was ejected and a new tip and nozzle were used. Each chip nozzle had an internal diameter of $2.5 \mu \mathrm{m}$, which under the given conditions, delivered a working flow rate of approximately $50 \mathrm{~nL} / \mathrm{min}$.

Applied ganglioside nomenclature

Gangliosides and the precursor glycosphingolipids are abbreviated according to the system introduced by Svennerholm in 1980 [30] and the recommendations of IUPAC-IUB Commission on Biochemical Nomenclature [34] as presented in Table 1 .

\section{Results and discussions}

MS comparative profiling of $\mathrm{HFC}$ and NFC ganglioside mixtures

Purified ganglioside mixtures extracted from normal and hemangioma bearing frontal lobe cortical tissue were

Table 1 Ganglioside nomenclature according to the systerns introduced by Svennerhom and IUPAC-IUB

\begin{tabular}{|c|c|}
\hline $\begin{array}{l}\text { Svennerholm } \\
\text { nomenclature }\end{array}$ & IUPAC-IUB nomenclature \\
\hline LacCer & Gal $\beta 4 \mathrm{Glc} \beta$ ICer \\
\hline $\mathrm{GA} 2, \mathrm{Gg}_{3} \mathrm{Cer}$ & 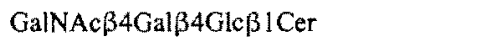 \\
\hline $\mathrm{GA}, \mathrm{Gg}_{4} \mathrm{Cer}$ & Gal $\beta 3$ GalNAc $\beta 4$ Gal $\beta 4 G l c \beta 1 \mathrm{Cer}$ \\
\hline $\mathrm{nLc} c_{4} \mathrm{Cer}$ & Gal $\beta 4 \mathrm{GlcNAc} \beta 3 \mathrm{Gal} \beta 4 \mathrm{Glc} \beta 1 \mathrm{Cer}$ \\
\hline $\mathrm{Lc}_{4} \mathrm{Cer}$ & Gal $\beta 3 \mathrm{GlcNAc} \beta 3 \mathrm{Gal} \beta 4 \mathrm{Glc} \beta 1 \mathrm{Cer}$ \\
\hline GM4 & $I^{3} \cdot \alpha-$ Neu 5 Ac-GlcCer \\
\hline GM3 & $11^{3}-\alpha-$ Neu 5 Ac-LacCer \\
\hline GD3 & $I^{3}-\alpha-(\mathrm{Neu} 5 \mathrm{Ac})_{2}-\mathrm{LacCer}$ \\
\hline GT3 & $11^{3}-\alpha-(\mathrm{Neu} S \mathrm{Ac})_{3}-\mathrm{LacCer}$ \\
\hline GM2 & $\mathrm{II}^{3}-\alpha-\mathrm{Neu} 5 \mathrm{Ac}-\mathrm{Gg}_{3} \mathrm{Cer}$ \\
\hline GD2 & $11^{3}-\alpha-(\mathrm{Neu} 5 \mathrm{Ac})_{2}-\mathrm{Gg}_{3} \mathrm{Cer}$ \\
\hline GMla & $\mathrm{II}^{3}-\alpha-\mathrm{Neu} 5 \mathrm{Ac}-\mathrm{Gg} \mathrm{g}_{4} \mathrm{Cer}$ \\
\hline GMIb & $\mathrm{IV}^{3}-\alpha-\mathrm{Neu} 5 \mathrm{Ac}-\mathrm{Gg}_{4} \mathrm{Cer}$ \\
\hline LMI & $I V^{3}-\alpha-N e u 5 A c-L c_{4}$ Cer \\
\hline GDla & $I V^{3}-\alpha-N e u 5 A c, I^{3}-\alpha-N e u 5 A c-G g$ Cer \\
\hline GDIb & $\mathrm{II}^{3}-\alpha-(\mathrm{Neu} 5 \mathrm{Ac})_{2}-\mathrm{Gg}_{4} \mathrm{Cer}$ \\
\hline LDI & $\mathrm{IV}^{3}-\alpha-(\mathrm{Neu} 5 \mathrm{Ac})_{2}-\mathrm{L} c_{4} \mathrm{Cer}$ \\
\hline GTla & $I V^{3}-\alpha-($ Neu $\left.5 A c)\right)_{2}, I^{3}-\alpha-N e u 5 A c-G g_{4}$ Cer \\
\hline GTIb & $1 \mathrm{~V}^{3}-\alpha-\mathrm{Neu} 5 \mathrm{Ac}, \mathrm{II}^{3}-\alpha-(\mathrm{Neu} 5 \mathrm{Ac})_{2}-\mathrm{Gg}{ }_{4} \mathrm{Cer}$ \\
\hline GTle & $1 I^{3}-\alpha-(\mathrm{Neu} 5 \mathrm{Ac})_{3}-\mathrm{Gg} \mathrm{g}_{4} \mathrm{Cer}$ \\
\hline GQIb & $\mathrm{IV}^{3}-\alpha-(\mathrm{Neu} 5 \mathrm{Ac})_{2}, \mathrm{II}^{3}-\alpha-(\mathrm{Neu} 5 \mathrm{Ac})_{2}-\mathrm{Gg}_{4} \mathrm{Cer}$ \\
\hline GQIc & $\mathrm{IV}^{3}-\alpha-\mathrm{Neu} 5 \mathrm{Ac}, \mathrm{II}^{3}-\alpha-(\mathrm{Neu} 5 \mathrm{Ac})_{3}-\mathrm{Gg}_{4} \mathrm{Cer}$ \\
\hline
\end{tabular}

submitted to high-throughput negative nanoESI chip $\mathrm{MS}^{1}$ screening under identical solution and instrumental parameters. The obtained mass spectra are presented in Figs. 1 and 2 while the ion assignments to ganglioside structures are listed in Tables 2 and 3, respectively. Each mass spectrum is a sum of 20 scans acquired within only $0.5 \mathrm{~min}$. The postulation of structures in Tables 2 and 3 was carried out by mass calculation and was based also on the previously described evidences, knowledge of this type of substrates [24] and biosynthesis pathway criteria.

General inspection of spectra shows that optimized nanoESI chip HCT MS conditions allowed for the concomitant formation of singly and doubly charged ions, enhanced the ionization of long chain polysialylated GT and GQ structures, provided a fair ionization/detection of minor components and minimized the in-source fragmentation of labile carbohydrate or non-carbohydrate type of modifications such as NeuSAc, $O-A c$ and terminal $O$-GalNAc. As visible, even under the employed timerestrictive high-throughput conditions, the acquired spectra feature a high signal-to-noise $(S / N)$ ratio and a remarkably rich molecular ion pattern.

Comparative examination of the spectra in Figs. 1 and 2 obtained under identical solution and instrumental conditions revealed first of all a significant difference in the number of ganglioside components expressed in hemangioma (Table 2) vs. normal tissue (Table 3). 43 ganglioside species were identified in NFC but only 29 in HFC.

NFC tissue appears to contain a higher diversity of ganglioside structures differing in their sialylation status, from short, monosialylated (GM) to large, polysialylated carbohydrate chains (GQ) and also ganglioside chains modified by $O$-Ac and GalNAc labile peripheral attachments. From a total of 43 species, 13 are monosialylated while 30 contain more than one sialic acid moiety attached to their oligosaccharide core. Thus, on the basis of their exact mass calculation, $11 \mathrm{di}-, 14$ tri- and five pentasialylated structures were identified. Nine of 14 trisialylated gangliosides exhibit a GT1-type of carbohydrate core linked to different ceramide forms. In the case of pentasialylated structures, all five are pentasialotetraoses (GQ1-type). Moreover, three biologically significant acylated gangliosides and one $O$-GalNAc-altered species were discovered as well. They represent $O$-acetylated variants of GM4 (d18:1/25:2) detected as a singly charged monodeprotonated ion at $\mathrm{m} / \mathrm{z}$ 1153.96, GM3 (d18:0/32:0) detected also as a singly charged monodeprotonated ion at $\mathrm{m} / \mathrm{z}$ 1419.91, GD2 (d18:1/23;0) corresponding to the singly charged monodeprotonated ion at $m / z 1786.16$ and $O$-GalNAc-modified GD1 (d:18:1/20:0) detected as a doubly deprotonated ion at $m / z 1033.81$.

Contrastingly, the ganglioside mixture extracted from HFC is essentially dominated by species of short oligosaccharide chains and exhibits reduced overall sialic acid 


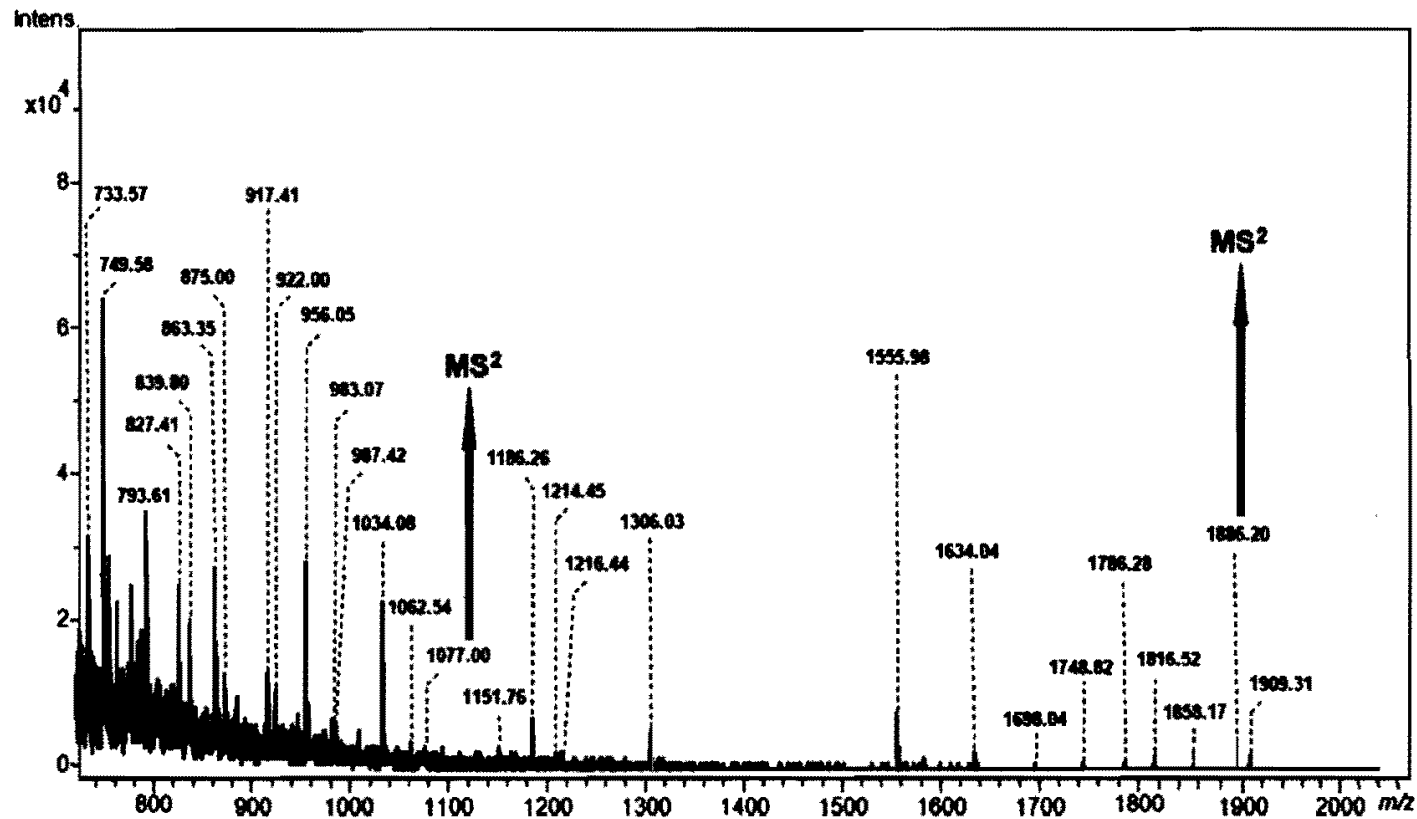

Fig. 1 Fully automated nanoESI chip HCT MS ${ }^{1}$ of the native HFC ganglioside mixture extracted from frontal lobe hemangioma tissue. Solvent, $\mathrm{MeOH}$; sample concentration $0.5 \mathrm{pmol} / \mu \mathrm{L}$; acquisition time $0.5 \mathrm{~min}$; chip-ESI, $-0.85 \mathrm{kV}$; capillary exit, $-30 \mathrm{~V}$

content. From a total of 29 structures identified in hemangioma tissue, 13 are monosialylated species of GMI, GM2, GM3, and GM4-type and 13 are disialylated species of GDI and GD2-type bearing ceramides of variable constitution.

In the spectrum in Fig. 1, only two GT ganglioside structures were detected: one GT1 having (d18:1/20:0) ceramide composition and one GT3 with (d18:1/25:1) ceramide. Interestingly, none of these trisialylated ganglioside forms were detected in NFC, which shows that these components are either absent or expressed in concentrations below the instrument detection limits in NFC. Therefore, they seem rather associated with HFC but not with NFC.

According to earlier studies, the expression of polysialylated gangliosides is regulated in a growth-and development-
Fig. 2 Fully automated nanoESI chip HCT MS ${ }^{l}$ of the native NFC ganglioside mixture extracted from normal frontal cortical tissue. Solvent, $\mathrm{MeOH}$; sample concentration $0.5 \mathrm{pmol} /$ $\mu \mathrm{L}$; acquisition time $0.5 \mathrm{~min}$; chip-ESI, $-0.85 \mathrm{kV}$; capillary exit, $-30 \mathrm{~V}$

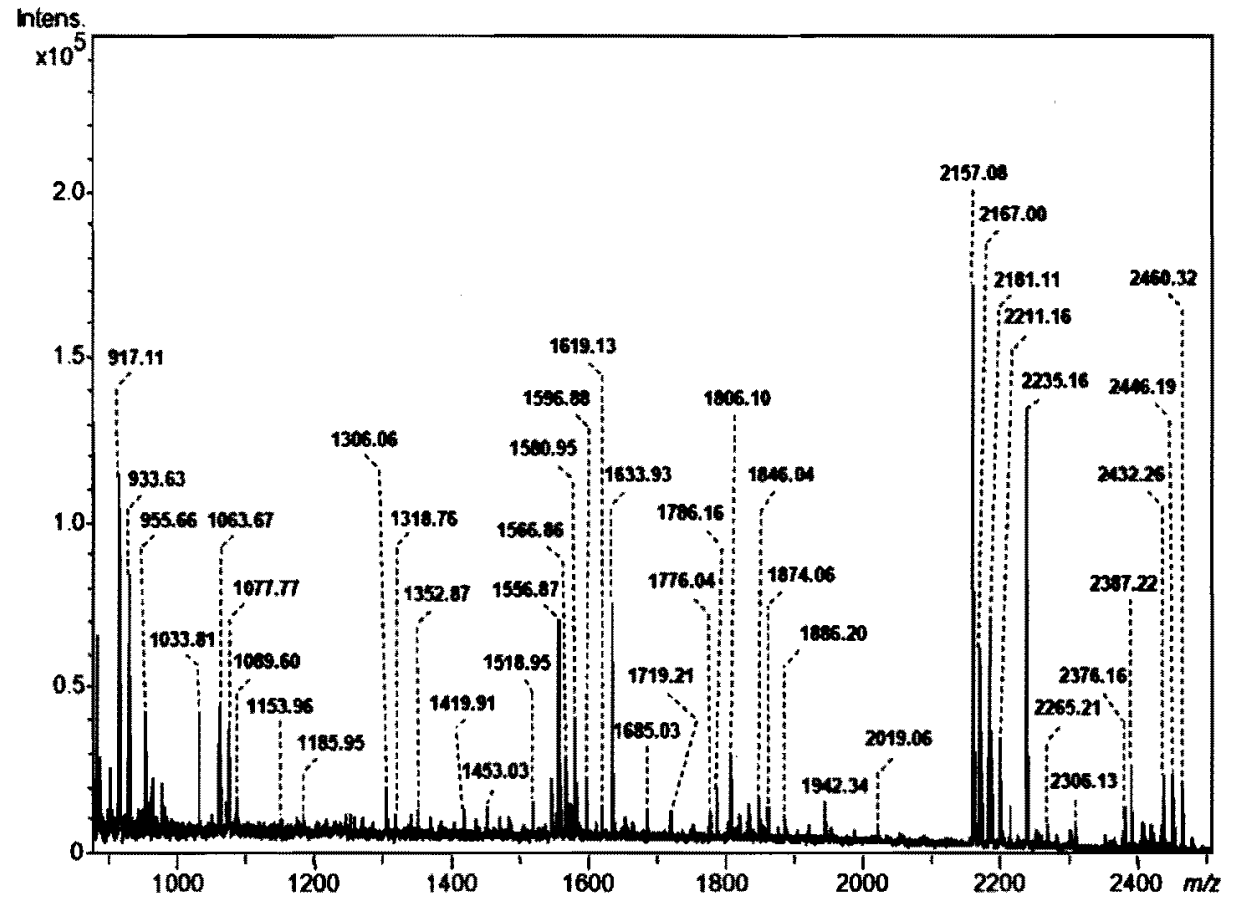


Table 2 Assignment of the major molecular ions detected in $\mathrm{HFC}$ ganglioside mixture

\begin{tabular}{|c|c|c|c|}
\hline Type of molecular ion & Experimental $m / z$ (monoisotopic) & Theoretic $m / z$ (monoisotopic) & Assigned structure \\
\hline$[\mathrm{M}-2 \mathrm{H}]^{2-}$ & 733.57 & 732.96 & GM2(di8:1/24:0) \\
\hline$[\mathrm{M}-2 \mathrm{H}]^{2-}\left(-2 \mathrm{H}_{2} \mathrm{O}\right)$ & 749.58 & 749.07 & GM2(d18:1/29:1) \\
\hline$[\mathrm{M}-\mathrm{H}]^{2-}\left(-\mathrm{H}_{2} \mathrm{O}\right)$ & 793.61 & 793.43 & GD2(d18:0/13:0) \\
\hline$[\mathrm{M}-\mathrm{H}]^{2-}\left(-\mathrm{H}_{2} \mathrm{O}\right)$ & 827.41 & 827.45 & $\mathrm{GD} 2(\mathrm{~d} 18: 1 / 18: 0)$ \\
\hline$[\mathrm{M}-\mathrm{H}]^{2-}\left(-\mathrm{H}_{2} \mathrm{O}\right)$ & 839.80 & 839.91 & GM1(d18:1/29:0) \\
\hline$[\mathrm{M}-\mathrm{H}]^{2-}$ & 863.35 & 863.49 & $\mathrm{GD} 2(\mathrm{~d} 18: 1 / 22: 1)$ \\
\hline$[\mathrm{M}+\mathrm{Na}-2 \mathrm{H}]^{2-}$ & 875.00 & 875.15 & GM1(d18:0/31:0) \\
\hline$[\mathrm{M}-\mathrm{H}]^{2-}$ & 917.41 & 917.49 & GDl(d18:1/18:0) \\
\hline$[\mathrm{M}+\mathrm{Na}-2 \mathrm{H}]^{2-}$ & 922.00 & 922.51 & GDI(d18:0/17:0) \\
\hline$[\mathrm{M}+\mathrm{Na}-2 \mathrm{H}]^{2-}$ & 956.05 & 956.51 & GDI(d18:1/22:0) \\
\hline$[\mathrm{M}-\mathrm{H}]^{-}$ & 983.07 & 983.61 & GM4(d18:1/16:3) \\
\hline$[\mathrm{M}-\mathrm{H}]$ & 987.42 & 987.64 & GM4(d18:1/16:1) \\
\hline$[\mathrm{M}-\mathrm{H}]$ & $1,034.08$ & $1,033.81$ & GM4(d18:0/19:0) \\
\hline$[\mathrm{M}-\mathrm{H}]^{-}$ & $1,062.54$ & $1,061.86$ & GM4(d|8:0/21:0) \\
\hline$[\mathrm{M}-2 \mathrm{H}]^{2-}$ & $1,077.00$ & $1,077.05$ & GTI(d18:1/20:0) \\
\hline$[\mathrm{M}-\mathrm{H}]^{-}$ & $1,151.76$ & $1,151.71$ & GM3(d|8:0/16:1) \\
\hline$[\mathrm{M}-\mathrm{H}]^{-}$ & $1,186.26$ & $1,185.78$ & GM3(d18:1/19:4) \\
\hline$[\mathrm{M}+2 \mathrm{Na}-3 \mathrm{H}]^{-}$ & $1,214.45$ & $1,214.00$ & GM4(d/8:1/29:1) \\
\hline$[\mathrm{M}-\mathrm{H}]^{-}$ & $1,216.44$ & $1,216.11$ & $O-A c-G M 4(\mathrm{~d} 18: 0 / 29: 0)$ \\
\hline$[\mathrm{M}-\mathrm{H}]^{-}$ & $1,306.03$ & $1,306.06$ & GM3(d18:1/27:0) \\
\hline$[\mathrm{M}-\mathrm{H}]\left(-2 \mathrm{H}_{2} \mathrm{O}\right)$ & $1,555.98$ & $1,555.81$ & GD2(d18:0/12:0) \\
\hline$[\mathrm{M}-\mathrm{H}]^{-}$ & $1,634.04$ & $1,633.93$ & GD2(18:0/15:0) \\
\hline$[\mathrm{M}-\mathrm{H}]\left(-\mathrm{H}_{2} \mathrm{O}\right)$ & $1,698.04$ & $1,698.06$ & GD2(d18:1/21:0) \\
\hline$[\mathrm{M}+3 \mathrm{Na}-4 \mathrm{H}]^{-}$ & $1,748.82$ & $1,748.96$ & $\operatorname{GM} 1(\mathrm{~d} 18: 1 / 28: 1)$ \\
\hline$[\mathrm{M}-\mathrm{H}]^{-}$ & $1,786.28$ & $1,786.16$ & O-Ac-GD2(d18:1/23:0) \\
\hline$[\mathrm{M}+\mathrm{Na}-2 \mathrm{H}]^{-}$ & $1,816.52$ & $1,816.17$ & GD2(d18:1/27:3) \\
\hline$[\mathrm{M}-\mathrm{H}]^{-}$ & $1,858.17$ & $1,858.18$ & GT3(d18:1/25:1) \\
\hline$[\mathrm{M}+\mathrm{Na}-2 \mathrm{H}]^{-}$ & $1,886.20$ & $1,885.99$ & GDI(d18:1/20:0) \\
\hline$[\mathrm{M}+2 \mathrm{Na}-3 \mathrm{H}]^{-}$ & $1,909.31$ & $1,909.98$ & GD2(d18:0/20:0) \\
\hline$[\mathrm{M}-2 \mathrm{H}]^{2-}$ & 733.57 & 732.96 & GM2(d18:1/24:0) \\
\hline$[\mathrm{M}-2 \mathrm{H}]^{2-}\left(-2 \mathrm{H}_{2} \mathrm{O}\right)$ & 749.58 & 749.07 & GM2(d18:1/29:1) \\
\hline$[\mathrm{M}-\mathrm{H}]^{2-}\left(-\mathrm{H}_{2} \mathrm{O}\right)$ & 73.61 & 793.43 & GD2(d18:0/13:0) \\
\hline$[\mathrm{M}-\mathrm{H}]^{2-}\left(-\mathrm{H}_{2} \mathrm{O}\right)$ & 827.41 & 827.45 & GD2(d18:1/18:0) \\
\hline$[\mathrm{M}-\mathrm{H}]^{2-}\left(-\mathrm{H}_{2} \mathrm{O}\right)$ & 839.80 & 839,91 & GMI(d18:1/29:0) \\
\hline$[\mathrm{M}-\mathrm{H}]^{2-}$ & 863.35 & 863.49 & GD2(d18:1/22:1) \\
\hline$[\mathrm{M}+\mathrm{Na}-2 \mathrm{H}]^{2-}$ & 875.00 & 875.15 & GM1(d18:0/31:0) \\
\hline$[\mathrm{M}-\mathrm{H}]^{2-}$ & 917.41 & 917.49 & GDI(d18:1/18:0) \\
\hline$[\mathrm{M}+\mathrm{Na}-2 \mathrm{H}]^{2-}$ & 922.00 & 922.51 & GD1(d18:0/17:0) \\
\hline$[\mathrm{M}+\mathrm{Na}-2 \mathrm{H}]^{2-}$ & 956.05 & 956.51 & GDI(d18:1/22:0) \\
\hline$[\mathrm{M}-\mathrm{H}]^{-}$ & 983.07 & 983.61 & GM4(d18:1/16:3) \\
\hline$[\mathrm{M}-\mathrm{H}]^{-}$ & 987.42 & 987.64 & GM4(d18:1/16:1) \\
\hline$[\mathrm{M}-\mathrm{H}]^{-}$ & $1,034.08$ & $1,033.81$ & GM4(d18:0/19:0) \\
\hline$[\mathrm{M}-\mathrm{H}]$ & $1,062.54$ & $1,061.86$ & GM4(d18:0/21:0) \\
\hline$[\mathrm{M}-2 \mathrm{H}]^{2-}$ & $1,077.00$ & $1,077.05$ & $\operatorname{GTI}(\mathrm{d} 18: 1 / 20: 0)$ \\
\hline$[\mathrm{M}-\mathrm{H}]^{-}$ & $1,151.76$ & $1,151.71$ & GM3(d18:0/16:1) \\
\hline$[\mathrm{M}-\mathrm{H}]^{-}$ & $1,186.26$ & $1,185.78$ & GM3(d18:1/19:4) \\
\hline$[\mathrm{M}+2 \mathrm{Na}-3 \mathrm{H}]^{-}$ & $1,214.45$ & $1,214.00$ & GM4(d] 8:1/29:1) \\
\hline$[\mathrm{M}-\mathrm{H}]^{-}$ & $1,216.44$ & $1,216.11$ & O.AC-GM4(d $18: 0 / 29: 0)$ \\
\hline$[\mathrm{M}-\mathrm{H}]^{-}$ & $1,306.03$ & $1,306.06$ & GM3(d18:1/27:0) \\
\hline
\end{tabular}


Table 2 (continued)

\begin{tabular}{|c|c|c|c|}
\hline Type of molecular ion & Experimental $\mathrm{m} / \mathrm{z}$ (monoisotopic) & Theoretic $m / z$ (monoisotopic) & Assigned structure \\
\hline$[\mathrm{M}-\mathrm{H}]^{-}\left(-2 \mathrm{H}_{2} \mathrm{O}\right)$ & $1,555.98$ & $1,555.81$ & GD2(d18:0/12:0) \\
\hline$[\mathrm{M}-\mathrm{H}]^{-}$ & $1,634.04$ & $1,633.93$ & GD2(18:0/15:0) \\
\hline$[\mathrm{M}-\mathrm{H}]-\left(-\mathrm{H}_{2} \mathrm{O}\right)$ & $1,698.04$ & $1,698.06$ & GD2(d18:1/2l:0) \\
\hline$[\mathrm{M}+3 \mathrm{Na} 4 \mathrm{H}]^{-}$ & $1,748.82$ & $1,748.96$ & GM1(d18:1/28:1) \\
\hline$[\mathrm{M}-\mathrm{H}]^{-}$ & $1,786.28$ & $1,786.16$ & $O-A c-G D 2(\mathrm{~d} 18: 1 / 23: 0)$ \\
\hline$[\mathrm{M}+\mathrm{Na}-2 \mathrm{H}]^{-}$ & $1,816.52$ & $1,816.17$ & GD2(d18:1/27:3) \\
\hline$[\mathrm{M}-\mathrm{H}]^{-}$ & $1,858.17$ & $1,858.18$ & GT3(d18:1/25:1) \\
\hline$[\mathrm{M}+\mathrm{Na}-2 \mathrm{H}]^{-}$ & $1,886.20$ & $1,885.99$ & GD1(d $8: 1 / 20: 0)$ \\
\hline$[\mathrm{M}+2 \mathrm{Na}-3 \mathrm{H}]^{-}$ & $1,909.31$ & $1,909,98$ & $\mathrm{GD} 2(\mathrm{~d} 18: 0 / 20: 0)$ \\
\hline$[\mathrm{M}-2 \mathrm{H}]^{2-}$ & 733.57 & 732.96 & GM2(d18:1/24:0) \\
\hline$\left[\mathrm{M}-2 \mathrm{H}_{2} \mathrm{O}-2 \mathrm{H}\right]^{2-}$ & 749.58 & 749.07 & GM2(d18:1/29:1) \\
\hline$\left[\mathrm{M}-\mathrm{H}_{2} \mathrm{O}-\mathrm{H}\right]^{2-}$ & 793.61 & 793.43 & GD2(d18:0/13:0) \\
\hline$\left[\mathrm{M}-\mathrm{H}_{2} \mathrm{O}-\mathrm{H}\right]^{2-}$ & 827.41 & 827.45 & $\mathrm{GD} 2(\mathrm{~d} 18: 1 / 18: 0)$ \\
\hline$\left[\mathrm{M}-\mathrm{H}_{2} \mathrm{O}-\mathrm{H}\right]^{2-}$ & 839.80 & 839.91 & GMI(d18:1/29:0) \\
\hline$[\mathrm{M}-\mathrm{H}]^{2-}$ & 863.35 & 863.49 & $\mathrm{GD} 2(\mathrm{~d} 18: 1 / 22: 1)$ \\
\hline$[\mathrm{M}+\mathrm{Na}-2 \mathrm{H}]^{2-}$ & 875.00 & 875.15 & $\operatorname{GMl}(d 18: 0 / 31: 0)$ \\
\hline$[\mathrm{M}-\mathrm{H}]^{2-}$ & 917.41 & 917.49 & GD1(d18:1/18:0) \\
\hline$[\mathrm{M}+\mathrm{Na}-2 \mathrm{H}]^{2-}$ & 922.00 & 922.51 & GDI(d18:0/17:0) \\
\hline$[\mathrm{M}+\mathrm{Na}-2 \mathrm{H}]^{2-}$ & 956.05 & 956.51 & GD1(d18:1/22:0) \\
\hline$[\mathrm{M}-\mathrm{H}]^{-}$ & 983.07 & 983.61 & $\mathrm{GM} 4(\mathrm{~d} 18: 1 / 16: 3)$ \\
\hline$[\mathrm{M}-\mathrm{H}]^{-}$ & 987.42 & 987.64 & GM4(d18:1/16:1) \\
\hline$[\mathrm{M}-\mathrm{H}]^{-}$ & $1,034.08$ & $1,033.81$ & GM4(d18:0/19:0) \\
\hline$[\mathrm{M}-\mathrm{H}]^{-}$ & $1,062.54$ & $1,061.86$ & $\mathrm{GM} 4(\mathrm{~d} 18: 0 / 21: 0)$ \\
\hline$[\mathrm{M}-2 \mathrm{H}]^{2-}$ & $1,077.00$ & $1,077.05$ & GTI(d18:1/20:0) \\
\hline$[\mathrm{M}-\mathrm{H}]$ & $1,151.76$ & $1,151.71$ & GM3(d18:0/16:1) \\
\hline$[\mathrm{M}-\mathrm{H}]^{-}$ & $1,186.26$ & $1,185.78$ & GM3(d18:1/19:4) \\
\hline$[\mathrm{M}+2 \mathrm{Na}-3 \mathrm{H}]^{-}$ & $1,214.45$ & $1,214.00$ & GM4(d18:1/29:1) \\
\hline$[\mathrm{M}-\mathrm{H}]^{-}$ & $1,216.44$ & $1,216.11$ & $O-\mathrm{Ac}-\mathrm{GM} 4(\mathrm{~d} 18: 0 / 29: 0)$ \\
\hline$[\mathrm{M}-\mathrm{H}]^{-}$ & $1,306.03$ & $1,306,06$ & GM3(d18:1/27:0) \\
\hline$\left[\mathrm{M}-2 \mathrm{H}_{2} \mathrm{O}-\mathrm{H}\right]^{-}$ & $1,555.98$ & $1,555.81$ & GD2(d18:0/12:0) \\
\hline$[\mathrm{M}-\mathrm{H}]^{-}$ & $1,634.04$ & $1,633.93$ & $\mathrm{GD} 2(18: 0 / 15: 0)$ \\
\hline$\left[\mathrm{M}-\mathrm{H}_{2} \mathrm{O}-\mathrm{H}\right]$ & $1,698.04$ & $1,698.06$ & GD2(d18:1/21:0) \\
\hline$[\mathrm{M}+3 \mathrm{Na} \quad 4 \mathrm{H}]^{-}$ & $1,748.82$ & $1,748.96$ & GMI(d18:1/28:1) \\
\hline$[\mathrm{M}-\mathrm{H}]^{-}$ & $1,786.28$ & $1,786.16$ & $O-A c-G D 2(d 18: 1 / 23: 0)$ \\
\hline$[\mathrm{M}+\mathrm{Na}-2 \mathrm{H}]^{-}$ & $1,816.52$ & $1,816.17$ & GD2(d18:1/27:3) \\
\hline$[\mathrm{M}-\mathrm{H}]^{-}$ & $1,858.17$ & $1,858.18$ & GT3(d18:1/25:1) \\
\hline$[\mathrm{M}+\mathrm{Na}-2 \mathrm{H}]$ & $1,886.20$ & $1,885.99$ & GDI(d18:1/20:0) \\
\hline$[\mathrm{M}+2 \mathrm{Na}-3 \mathrm{H}]^{-}$ & $1,909.31$ & $1,909.98$ & $\mathrm{GD} 2(\mathrm{~d} 18: 0 / 20: 0)$ \\
\hline
\end{tabular}

dependent mode [35] and associated with the type of normal aberrant brain tissue status. For instance, it was previously reported that a lower content of sialylated species occurred in a gliosarcoma specimen in comparison with normal brain tissue [23]. Our present findings agree with the data collected by chip-ESI MS of brain gliosarcoma, and support the role of sialylation in tumor development and progression.

Unlike the previously investigated gliosarcoma [23], two modified ganglioside structures were observed in the ganglioside mixture extracted from $\mathrm{HFC}$. According to mass calculation, the $[\mathrm{M}-\mathrm{H}]^{-}$ion at $\mathrm{m} / \mathrm{z} 1,786.28$ is consistent with a structure of $O-A c-G D 2$ (d18:1/23:0) while $[\mathrm{M}-\mathrm{H}]^{-}$ion at $m / z \quad 1,216.44$ corresponds to $O$-AcGM4 (d18:0/29:0). The same O-Ac-GD2 (d18:1/23:0) species was identified also in the normal tissue of the frontal cortex, in higher abundance than in HFC, but could not be found in malignant tumors like gliosarcoma [23] which, lack any $O$-Ac-GD2 variants. This suggests that $O$ - 
Table 3 Assignment of the major molecular ions detected in NFC ganglioside mixture

\begin{tabular}{|c|c|c|c|}
\hline Type of Molecular lon & Experimental $m / z$ (monoisotopic) & Theoretic $m / z$ (monoisotopic) & Assigned structure \\
\hline$[\mathrm{M}-2 \mathrm{H}]^{2-}$ & 917.11 & 917.49 & GDI (d18:1/18:0) \\
\hline$\left[\mathrm{M}-2 \mathrm{H}_{2} \mathrm{O}-2 \mathrm{H}\right]^{2-}$ & 933.63 & 933.57 & GDI(d18:1/23:1) \\
\hline$[\mathrm{M}+\mathrm{Na}-3 \mathrm{H}]^{2-}$ & 955.66 & 955.50 & GDI(d $18: 1 / 22: 1)$ \\
\hline$[\mathrm{M}-2 \mathrm{H}]^{2-}$ & $1,033.81$ & $1,033.96$ & GalNAc-GD1(d18:1/20:0) \\
\hline$[\mathrm{M}-2 \mathrm{H}]^{2-}$ & $1,063.67$ & $1,063.04$ & GTI(d18:1/18:0) \\
\hline$[\mathrm{M}+2 \mathrm{Na}-3 \mathrm{H}]$ & $1,077.77$ & $1,077.77$ & GM4(d18:0/19:0) \\
\hline$[\mathrm{M}+\mathrm{Na}-3 \mathrm{H}]^{2-}$ & $1,089.60$ & $1,089.05$ & $\mathrm{GTl} 1 \mathrm{~d} 18: 0 / 20: 0)$ \\
\hline$[\mathrm{M}-\mathrm{H}]^{-}$ & $1,153.96$ & $1,153.96$ & $O-A c-G M 4(d 18: 1 / 25: 2)$ \\
\hline$[\mathrm{M}+2 \mathrm{Na}-3 \mathrm{H}]$ & $1,185.95$ & $1,185.78$ & GM4(d18:1/27:1) \\
\hline$[\mathrm{M}-\mathrm{H}]^{-}$ & $1,306.06$ & $1,306.06$ & $\operatorname{GM} 3(\mathrm{~d} \mid 8: 1 / 27: 0)$ \\
\hline$\left[\mathrm{M}-2 \mathrm{H}_{2} \mathrm{O}-\mathrm{H}\right]$ & $1,318.76$ & $1,318,76$ & GM2(d18:1/16:0) \\
\hline$[\mathrm{M}-\mathrm{H}]^{-}$ & $1,352.87$ & $1,352.78$ & GM2(d18:1/16:1) \\
\hline$[\mathrm{M}-\mathrm{H}]^{-}$ & $1,419.91$ & $1,420.02$ & O-Ac-GM3(d18:0/32:0) \\
\hline$[\mathrm{M}-\mathrm{H}]-$ & $1,453.03$ & $1,453.03$ & $\mathrm{GM} 2(\mathrm{~d} 18: 1 / 23: 0)$ \\
\hline$[\mathrm{M}-\mathrm{H}]$ & $1,518.95$ & $1,518.86$ & GM1(d18:0/16:0) \\
\hline$[\mathrm{M}-\mathrm{H}]-$ & $1,556.87$ & $1,556.96$ & GM1(d18:1/19:1) \\
\hline$[\mathrm{M}+\mathrm{Na}-2 \mathrm{H}]^{-}$ & $1,566.86$ & $1,566.86$ & GM1(d18:1/18:0) \\
\hline$[\mathrm{M}-\mathrm{H}]$ & $1,580.95$ & $1,580.95$ & GD3(d18:1/26:1) \\
\hline$[\mathrm{M}+2 \mathrm{Na}-3 \mathrm{H}]^{-}$ & $1,596.88$ & $1,596.88$ & GD3(d18:1/24:1) \\
\hline$[\mathrm{M}+\mathrm{Na}-2 \mathrm{H}]$ & $1,619.13$ & $1,619.13$ & $\mathrm{GD} 3(\mathrm{~d} 18: 1 / 27: 0)$ \\
\hline$[\mathrm{M}-\mathrm{H}]^{-}$ & $1,633.93$ & $1,633.93$ & GD2(d18:0/15:0) \\
\hline$[\mathrm{M}-\mathrm{H}]^{-}$ & $1,685.03$ & $1,685.03$ & GMl(d18:1/28:0) \\
\hline$[\mathrm{M}+\mathrm{Na}-2 \mathrm{H}]$ & $1,719.21$ & $1,719.21$ & GMI(d18:1/29:1) \\
\hline$[\mathrm{M}-\mathrm{H}]^{-}$ & $1,776.04$ & $1,776.04$ & GT3(d18:1/19:0) \\
\hline$[\mathrm{M}-\mathrm{H}]^{-}$ & $1,786.16$ & $1,786.16$ & $O-\mathrm{Ac}-\mathrm{GD} 2(\mathrm{~d} 18: 1 / 23: 0)$ \\
\hline$[\mathrm{M}-\mathrm{H}]^{-}$ & $1,806.10$ & $1,806.10$ & GT3(d18:0/21:0) \\
\hline$[\mathrm{M}-\mathrm{H}]^{-}$ & $1,846.04$ & $1,846.04$ & GDI(d18:1/19:2) \\
\hline$[\mathrm{M}-\mathrm{H}]^{-}$ & $1,874.06$ & $1,874.06$ & GT3(d18:1/26:0) \\
\hline$[\mathrm{M}+\mathrm{Na}-2 \mathrm{H}]$ & $1,886.00$ & $1,885.99$ & GDl(d18:1/20:0) \\
\hline$[\mathrm{M}-\mathrm{H}]^{-}$ & $1,942.34$ & $1,942.34$ & GT3(d18:1/31:1) \\
\hline$[\mathrm{M}-\mathrm{H}]$ & $2,019.06$ & $2,019.06$ & GT2(d18:1/22:1) \\
\hline$[\mathrm{M}+2 \mathrm{Na}-3 \mathrm{H}]^{-}$ & $2,157.08$ & $2,157,08$ & GTI(d18:1/17:0) \\
\hline$[\mathrm{M}+2 \mathrm{Na}-3 \mathrm{H}]$ & $2,167.00$ & $2,167.00$ & GTl(d18:1/18:2) \\
\hline$[\mathrm{M}-\mathrm{H}]$ & $2,181.11$ & $2,181.11$ & GTI(d18:1/22:1) \\
\hline$[\mathrm{M}-\mathrm{H}]^{-}$ & $2,211.16$ & $2,211.16$ & GTI(d18:1/24:0) \\
\hline$[\mathrm{M}-\mathrm{H}]^{-}$ & $2,235.16$ & $2,235.16$ & GTl(d18:1/26:2) \\
\hline$[\mathrm{M}-\mathrm{H}]^{-}$ & $2,265.21$ & $2,265.21$ & GT1(d18:1/28:1) \\
\hline$[\mathrm{M}-\mathrm{H}]^{-}$ & $2,306.13$ & $2,306.04$ & GQI(d18:1/10:0) \\
\hline$[\mathrm{M}-\mathrm{H}]^{-}$ & $2,376.16$ & $2,376.16$ & GQ1(d18:1/15:0) \\
\hline$\left[\mathrm{M}+3 \mathrm{Na}-4 \mathrm{H}^{-}\right.$ & $2,387,22$ & $2,387.22$ & GT1(18:1/32:1) \\
\hline$[\mathrm{M}-\mathrm{H}]^{-}$ & $2,432.26$ & $2,432.26$ & GQI(d18:1/19:0) \\
\hline$[\mathrm{M}-\mathrm{H}]^{-}$ & $2,446.19$ & $2,446.19$ & GQ1(d18:1/20:0) \\
\hline$[\mathrm{M}-\mathrm{H}]^{-}$ & $2,460.32$ & $2,460.32$ & $\mathrm{GQI}(\mathrm{d} 18: 1 / 21: 0)$ \\
\hline$[\mathrm{M}-2 \mathrm{H}]^{2-}$ & 917.11 & 917.49 & GDI(d18:1/18:0) \\
\hline$[\mathrm{M}-2 \mathrm{H}]^{2-}\left(-2 \mathrm{H}_{2} \mathrm{O}\right)$ & 933.63 & 933.57 & GDI(dI8:1/23:1) \\
\hline$[\mathrm{M}+\mathrm{Na}-3 \mathrm{H}]^{2-}$ & 955.66 & 955.50 & $\operatorname{GDl}(\mathrm{d} 18: 1 / 22: 1)$ \\
\hline$[\mathrm{M}-2 \mathrm{H}]^{2-}$ & $1,033.81$ & $1,033.96$ & GalNAc-GDI(d1 8:1/20:0) \\
\hline$[\mathrm{M}-2 \mathrm{H}]^{2-}$ & $1,063.67$ & $1,063.04$ & GTI(dI8:1/18:0) \\
\hline$[\mathrm{M}+2 \mathrm{Na}-3 \mathrm{H}]^{-}$ & 1.077 .77 & $1,077.77$ & GM4(d18:0/19:0) \\
\hline
\end{tabular}


Table 3 (continued)

\begin{tabular}{|c|c|c|c|}
\hline Type of Molecular lon & Experimental $m / z$ (monoisolopic) & Theoretic $m / z$ (monoisotopic) & Assigned structure \\
\hline$[\mathrm{M}+\mathrm{Na}-3 \mathrm{H}]^{2-}$ & $1,089.60$ & $1,089.05$ & GT1 $(d 18: 0 / 20: 0)$ \\
\hline$[\mathrm{M}-\mathrm{H}]^{-}$ & $1,153.96$ & $1,153.96$ & O-Ac-GM4(dI8:1/25:2) \\
\hline$[\mathrm{M}+2 \mathrm{Na}-3 \mathrm{H}]$ & $1,185.95$ & $1,185.78$ & $\mathrm{GM} 4(\mathrm{~d} 18: 1 / 27: 1)$ \\
\hline$[\mathrm{M}-\mathrm{H}]^{-}$ & $1,306,06$ & $1,306.06$ & GM3(d18:1/27:0) \\
\hline$[\mathrm{M}-\mathrm{H}]-\left(-2 \mathrm{H}_{2} \mathrm{O}\right)$ & $1,318.76$ & $1,318.76$ & GM2(d $18: 1 / 16: 0)$ \\
\hline$[\mathrm{M}-\mathrm{H}]^{-}$ & $1,352.87$ & $1,352.78$ & GM2(d18:1/16:1) \\
\hline$[\mathrm{M}-\mathrm{H}]$ & $1,419.91$ & $1,420.02$ & O-Ac-GM3(d18:0/32:0) \\
\hline$[\mathrm{M}-\mathrm{H}]-$ & $1,453,03$ & $1,453.03$ & GM2(d18:1/23:0) \\
\hline$[\mathrm{M}-\mathrm{H}]$ & $1,518.95$ & $1,518.86$ & $\mathrm{GM} 1(\mathrm{~d} 18 ; 0 / 16: 0)$ \\
\hline$[\mathrm{M}-\mathrm{H}]-$ & $1,556.87$ & $1,556.96$ & GMl(d18:1/19:1) \\
\hline$[\mathrm{M}+\mathrm{Na}-2 \mathrm{H}]^{-}$ & $1,566.86$ & $1,566.86$ & GMI(d18:1/18:0) \\
\hline$[\mathrm{M}-\mathrm{H}]-$ & $1,580.95$ & $1,580.95$ & GD3(d18:1/26:1) \\
\hline$[\mathrm{M}+2 \mathrm{Na}-3 \mathrm{H}]^{-}$ & $1,596.88$ & $1,596.88$ & GD3(d1 8:1/24:1) \\
\hline$[\mathrm{M}+\mathrm{Na}-2 \mathrm{H}]^{-}$ & $1,619.13$ & $1,619,13$ & GD3(d1 8:1/27:0) \\
\hline$[\mathrm{M}-\mathrm{H}]^{-}$ & $1,633,93$ & $1,633.93$ & GD2(d18:0/15:0) \\
\hline$[\mathrm{M}-\mathrm{H}]^{-}$ & $1,685.03$ & $1,685.03$ & $\operatorname{GMI}(\mathrm{d} \mid 8: 1 / 28: 0)$ \\
\hline$[\mathrm{M}+\mathrm{Na}-2 \mathrm{H}]^{-}$ & $1,719.21$ & $1,719.21$ & GMI(dI8:1/29:1) \\
\hline$[\mathrm{M}-\mathrm{H}]$ & $1,776,04$ & $1,776.04$ & GT3(d18:1/19:0) \\
\hline$[\mathrm{M}-\mathrm{H}]^{-}$ & $1,786.16$ & $1,786.16$ & O-Ac-GD2(d18:1/23:0) \\
\hline$[\mathrm{M}-\mathrm{H}]^{-}$ & $1,806.10$ & $1,806.10$ & GT3(d18:0/21:0) \\
\hline$[\mathrm{M}-\mathrm{H}]^{-}$ & $1,846,04$ & $1,846.04$ & GD1(d18:1/19:2) \\
\hline$[\mathrm{M}-\mathrm{H}]^{-}$ & $1,874,06$ & $1,874.06$ & GT3(d18:1/26;0) \\
\hline$[\mathrm{M}+\mathrm{Na}-2 \mathrm{H}]^{-}$ & $1,886.00$ & $1,885.99$ & GDI(d18:1/20:0) \\
\hline$[\mathrm{M}-\mathrm{H}]^{-}$ & $1,942.34$ & $1,942.34$ & GT3(d18:1/31:1) \\
\hline$[\mathrm{M}-\mathrm{H}]^{-}$ & $2,019.06$ & $2,019.06$ & Gr2(d18:1/22:1) \\
\hline$[\mathrm{M}+2 \mathrm{Na}-3 \mathrm{H}]$ & $2,157.08$ & $2,157.08$ & GT1(d18:1/17:0) \\
\hline$[\mathrm{M}+2 \mathrm{Na}-3 \mathrm{H}]$ & $2,167.00$ & $2,167.00$ & GTI(d18:1/18:2) \\
\hline$[\mathrm{M}-\mathrm{H}]^{-}$ & $2,181.11$ & $2,181,11$ & GT1(d18:1/22:1) \\
\hline$[\mathrm{M}-\mathrm{H}]^{-}$ & $2,211.16$ & $2,211.16$ & GTI(dl8:1/24:0) \\
\hline$[\mathrm{M}-\mathrm{H}]^{-}$ & $2,235.16$ & $2,235.16$ & GT1(dI8:1/26:2) \\
\hline$[\mathrm{M}-\mathrm{H}]^{-}$ & $2,265.21$ & $2,265.21$ & $\operatorname{GTl}(\mathrm{d} 18: 1 / 28: 1)$ \\
\hline$[\mathrm{M}-\mathrm{H}]^{-}$ & $2,306.13$ & $2,306.04$ & GQI(d18:1/10:0) \\
\hline$[\mathrm{M}-\mathrm{H}]^{-}$ & $2,376.16$ & $2,376.16$ & GQI(d18:1/15:0) \\
\hline$[\mathrm{M}+3 \mathrm{Na}-4 \mathrm{H}]$ & $2,387.22$ & $2,387.22$ & GT1(18:1/32:1) \\
\hline$[\mathrm{M}-\mathrm{H}]^{-}$ & $2,432.26$ & $2,432.26$ & GQI(d18:1/19:0) \\
\hline$[\mathrm{M}-\mathrm{H}]^{-}$ & $2,446.19$ & $2,446.19$ & GQI(d18:1/20:0) \\
\hline$[\mathrm{M}-\mathrm{H}]^{-}$ & $2,460.32$ & $2,460.32$ & GQI(d18:1/21:0) \\
\hline
\end{tabular}

Ac-GD2 structures might be markers for cerebral tumors of reduced malignancy grade.

CID $\mathrm{MS}^{2}$ fragmentation analysis of the polysialylated HFC-associated ganglioside species

The low abundant doubly charged ion at $m / z \quad 1,077.00$ which, according to mass calculation corresponds to GT1 (d18:0/20:0), was isolated within an isolation width of $2 u$ and submitted to fragmentation analysis by CID $\mathrm{MS}^{2}$.
Obtained tandem mass spectrum is presented in Fig. 3a while the scheme illustrating the most probable fragmentation pathways, along with the designation of product ions supporting the sequencing processes discussed below is shown in Fig. 3b. As visible in Fig. 3a, even for such low intensity precursor ions, HCT CID $\mathrm{MS}^{2}$ exhibits a remarkably high signal-to-noise ratio and nearly nil baseline noise level. This beneficial feature of ion sequencing in HCT analyzer by either CID, electron transfer dissociation (ETD) or alternate CID/ETD, often reported 


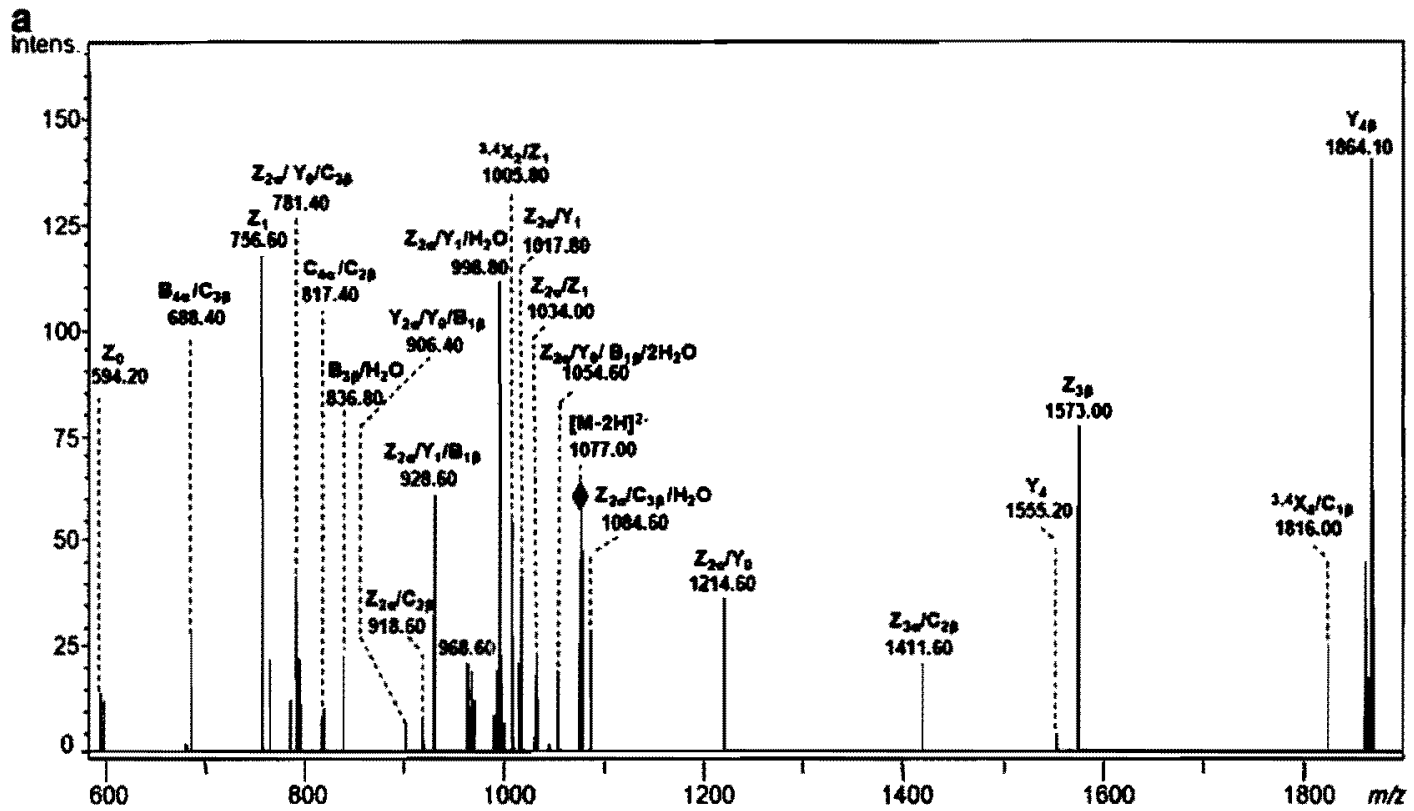

b

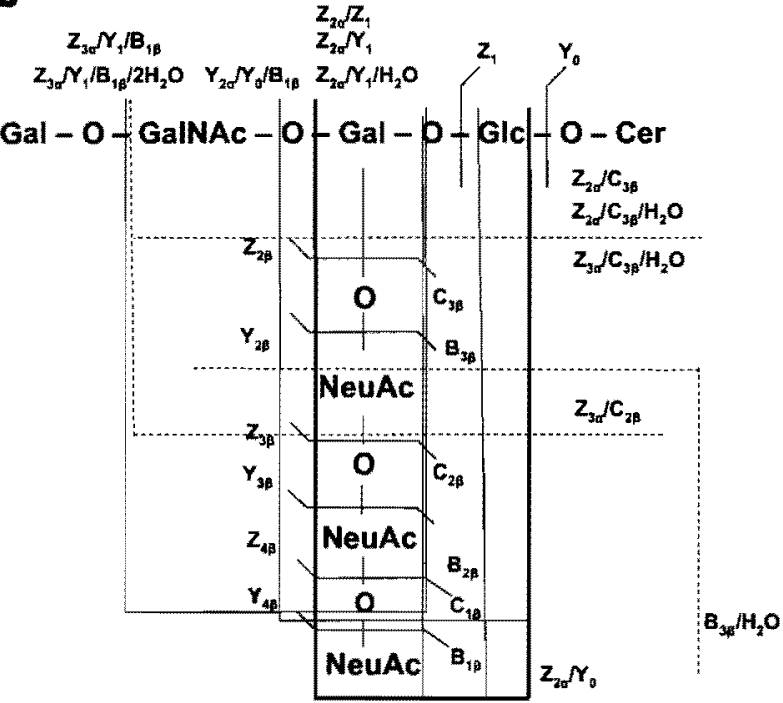

Fig. 3 Fragmentation analysis of the $[\mathrm{M}-2 \mathrm{H}]^{2-}$ at $m / z \quad 1,077.00$ corresponding to GTI(18:1/20:0) ganglioside species detected in HFC ganglioside mixture. a Fully automated nanoESI chip HCT MS'; b

lately $[26-29,36-39]$, demonstrates the gain in sensitivity provided by the new generation of ion trap mass spectrometers of high ion-storage capacity.

Spectrum evaluation shows that by employing ion fragmentation at variable excitation amplitudes, a high product ion yield and generation of ions diagnostic for certain GT1 (d 18:0/20:0) isomers were obtained. Moreover, lack of extensive molecule desialylation observed in the spectnum in Fig. $3 a$ is of critical importance for the assessment of molecule sialylation status and sites. The most interesting aspect revealed by the spectrum in Fig. $3 \mathrm{a}$ is the presence of fragmentation scheme corresponding to the GTIc isomer. CID at variable of signal amplitudes within $0.6-1.0 \mathrm{~V}$. Acquisition time $0.5 \mathrm{~min}$ uncommon GT1c isomer in the ganglioside mixture extracted from HFC. Five different fragment ions at $\mathrm{m} / \mathrm{z}$ $836.80,998.80,1017.80,1034.00$, and 1214.60 , all singly charged monodeprotonated, support a structure of GT Ic-type bearing ( $\mathrm{d} 18: 0 / 20: 0$ ) ceramide also documented by $\mathrm{Z}_{0}{ }^{-}$ion at $m / z 594.20$. According to mass calculation, the ion at $m / z$ 836.80 corresponds to the dehydrated trisialo element showing that three Neu5Ac residues are linked together, a configuration specific to either GT1c or GT1d glycoforms. These two structures are supported also by the ions at $m / z$ $1,017.80$ assigned to $\mathrm{Z}_{2 \alpha} / Y_{1}^{-}, m / z 998.80$ assigned to 
dehydrated $\mathrm{Z}_{2 \alpha} / \mathrm{Y}_{1}^{-}$and $\mathrm{m} / \mathrm{z} \quad 1,034,00$ corresponding to $\mathrm{Z}_{2 \alpha} / \mathrm{Y}_{1}^{-}$, all having the composition (GalNeu5 $\left.\mathrm{Ac}_{3}\right)^{-}$. However, the detection of the fairly abundant $\mathrm{Z}_{2 \alpha} / \mathrm{Y}_{0}{ }^{-}$ ion at $m / z \quad 1,214.60$ is indubitable evidence regarding the occurrence of GTlc isomer. This ion, having the composition (GlcGalNeuS $\left.\mathrm{Ac}_{3}\right)^{-}$, demonstrates that in some of the simultaneously isolated and sequenced isomers/isobars, trisialo element is definitely connected to the inner galactose residue, a configuration consistent with GTlc form. On the other hand, the ion at $m / z 906.40$ assigned to $Y_{2 \alpha} / Y_{\alpha} / B_{1 \beta}{ }^{-}$ corroborates the presence of both GTlc and GTlb species (Fig. 3b). Because of the Gal-GalNAc-Gal chain symmetry the structure corresponding to GTla and GTld cannot be discriminated via specific diagnostic ions. However, the occurrence of these isoforms cannot be excluded. Consequently, we suspect that all four GT1a, GT1b, GT1c, and GTld isomers are present in the hemangioma mixture; however, because the presence of GTlc is supported by the highest number of diagnostic ions, we have chosen to depict in the scheme in Fig. 3b, the fragmentation pathway experienced by the ion corresponding to this isomer.

To confirm this concept discussed above, the singly charged monodeprotonated sodiated ion detected in the HFC ganglioside mixture spectrum at $\mathrm{m} / \mathrm{z} 1,886.20$ was submitted to CID $\mathrm{MS}^{2}$ under identical sequencing conditions. According to mass calculation, this ion corresponds

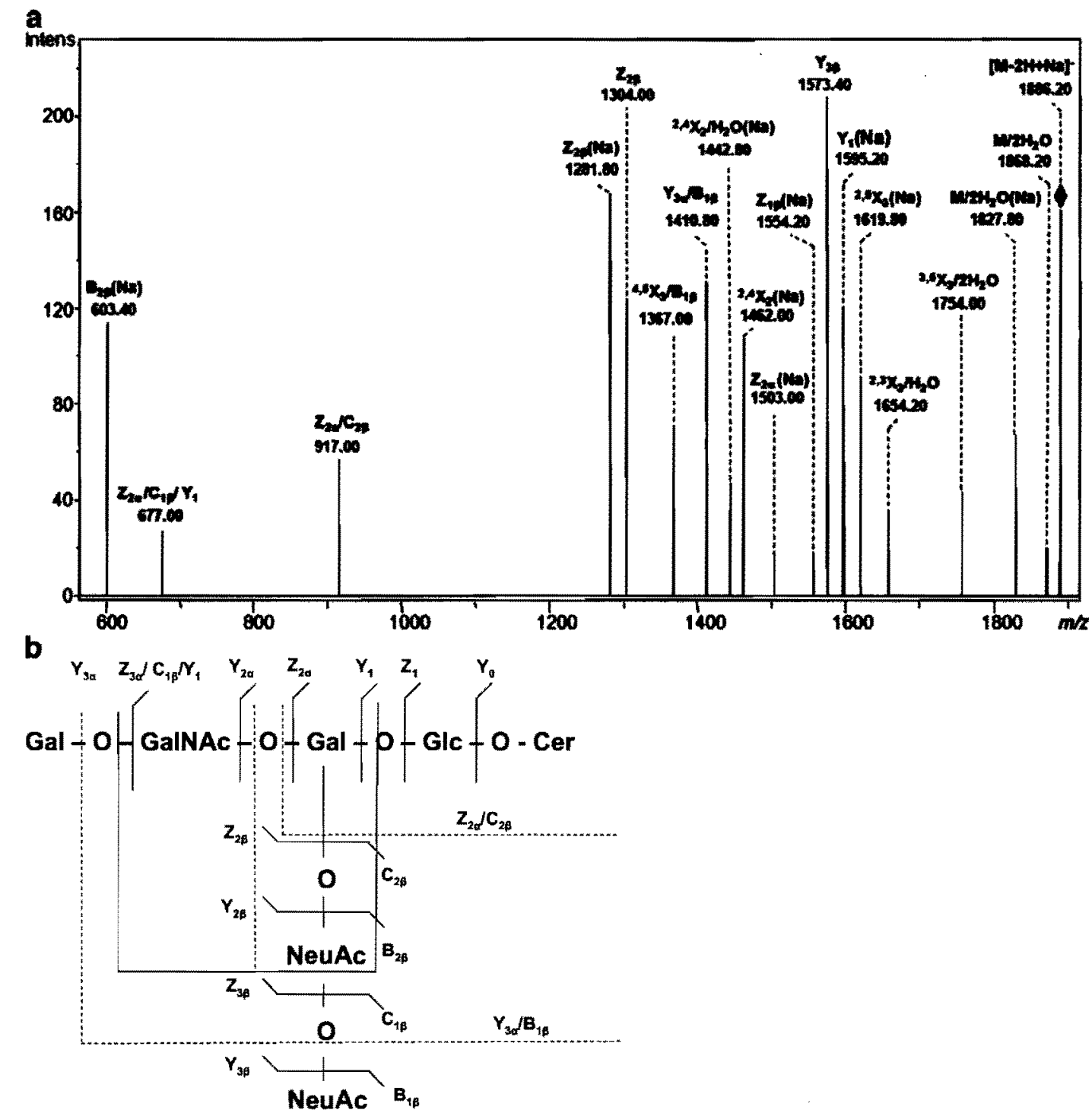

Fig. 4 Fragmentation analysis of the $[\mathrm{M}-2 \mathrm{H}+\mathrm{Na}\rceil$ at $m / z \quad 1,886.20$ corresponding GDI(18:1/20:0) ganglioside species detected in HFC ganglioside mixture. a Fully automated nanoESI chip HCT $\mathrm{MS}^{2}$; b fragmentation scheme corresponding to the GDIb isomer. CID at variable of signal amplitudes within $0.6-1.0 \mathrm{~V}$. Acquisition time $0.5 \min$ 
to a GDl structure having the same (d18:0/20:0) lipid part constitution of the previously sequenced GT1 but with one less Neu5Ac residue. CID $\mathrm{MS}^{2}$ spectrum is presented in Fig. $4 \mathrm{a}$ while the fragmentation scheme corresponding to GDIb isomer is shown in Fig. 4b. Disialo element detected as sodiated $\mathrm{B}_{2 \beta}{ }^{-}$ion at $m / z 603.40$ is a strong indication of the presence of GDIb (d18:0/20:0) isomer in HFC. This molecular structure exhibiting both sialic acids linked to the inner galactose is also documented by the ion at $\mathrm{m} / \mathrm{z}$ 1,503.00 assigned to $\mathrm{Z}_{2 \mathrm{a}}{ }^{-}(\mathrm{Na})$ having the composition (NeuSAc $\mathrm{AalGlcCer}_{2}{ }^{2}$. Similarly, since no ion exclusively diagnostic for GDla was detected, the presence of this type of isomer cannot be excluded.

These similarities in fragmentation pathways suggest that GDlb (d18:0/20:0) could have been subject to tumoral transformation in GTlc (d18:0/20:0) via galactosyltransferase II (Gal T-II) [40] or GT1c could have lost a sialic acid during the tissue alteration processes.

\section{Conclusions}

We developed here a modern methodology for the investigation of ganglioside expression and structure in a benign human brain tumor. According to our knowledge, this is the first preliminary study of gangliosides in a histopathologically defined hemangioma brain tumor and, certainly, the first utilizing the advanced mass spectrometric method based on fully automated chip-nanoESI directly coupled to HCT MS and CID MS ${ }^{2}$.

The ganglioside composition in hemangioma was found highly altered in comparison to the ganglioside expression in normal human brain. While in $\mathrm{HFC}$, short, monosialylated gangliosides prevail, in NFC mono- and polysialylated gangliosides as well as $O$-acetylated components were found as major species. We assume that this altered pattern in hemangioma vs. healthy control tissue is the result of a different overall biosynthetic rate, due to the change in expression of certain glycosyltransferases. Nevertheless, in HFC, we were still able to detect one O-Ac-GM4 and one $O$ Ac-GD2 species and correlate the presence of $O$-Ac-GD2 with the reduced malignancy grade of the investigated cerebral tumor. Additionally, a GT1 (d18:0/20:0) detected as a low intensity ion, appeared to be another marker found only in HFC. CID $\mathrm{MS}^{2}$ on the GT1 (d18:0/20:0)corresponding molecular ion provided evidence of the existence of the less common GTlc isomer. This interesting structural feature was further supported by the CID $\mathrm{MS}^{2}$ analysis of GDI (d18:0/20:0)-related ion.

Finally, a notable aspect is the reproducibility, speed, and sensitivity of the analysis. Under the same experimental conditions, the method provided almost $100 \%$ nun-to-run and in-nun reproducibility of the obtained data. Since the robotic system sprayed with a flow rate of $50 \mathrm{~nL} / \mathrm{min}$ and considering a sample concentration of $0.5 \mathrm{pmol} / \mu \mathrm{L}$, 1.5 min signal acquisition of one HFC screening MS and two CID $\mathrm{MS}^{2}$ resulted in a sample consumption below 40 fmols. To our knowledge, this is the highest sensitivity reported so far in ganglioside analysis by electrospray ionization mass spectrometry.

These initial results indicate the feasibility of ultrasensitive and accurate mass spectrometric methods in determining an altered composition of brain gangliosides. Due to the high sensitivity for detection and sequencing of minor carbohydrate species in complex mixtures, speed of analysis, and other here-demonstrated advantages, the chipbased nanoESI HCT MS and CID MS ${ }^{n}$ has the potential to be introduced in medical diagnostics for comparative compositional and structural analysis of homologous biological glycoconjugates from either tissues or body fluids. Therefore, full consideration is currently given to further system refinement, on-line combination with liquid chromatography and capillary electrophoresis for implementation in clinical investigations focused on discovery of specific biochemical markers useful in early diagnosis and therapy of benign and malignant proliferations.

Acknowledgments This work was supported by Romanian National Authority for Scientific Research through the PN-II-41001/2007 project. Permission for experiments with human tissue for scientific purposes was obtained from the Ethical Commission of "Victor Babes" University of Medicine and Pharmacy Timisoara and Zagreb Medical School, under the Project no. 108120 granted by the Croatian Ministry of Science and Technology.

\section{References}

1. Sicgel AM (1998) Acta Neurol Scand 98:369-371

2. Pozzati E, Acciarri N, Tognetti F, Marliani F, Giangaspero F (1996) Neurosurgery $38: 662-670$

3. Houtteville JP (1997) Surg Neurol 48:610-614

4. Detweiler PW, Porter RW, Zabramski JM, Spetzler RF (1997) J Neurosurg 87:629-632

5. Dorner L, Buhl R, Hugo HH, Jansen O, Barth H, Mehdom HM (2005) Acta Ncurochir 147:1091-1096

6. Mosnier JF, Brunon J, Nuti C (2007) Neurochirurgie 53:131-135

7. Lehnhardt FG, von Smekal U, Rückriem B, Stenzel W, Neveling M, Heiss WD, Jacobs AH (2005) Arch Neurol 62:653-658

8. Ledeen RW, Yu RK (1982) Methods Enzymol 83:139 191

9. Fishman PH, Brady RO (1976) Science 194:906-915

10. Ledeen RW J (1978) Supramol Struct 8:1-17

11. Ledeen RW, Yu RK (1982) Methods Enzymol 83:109-191

12. Yu RK, Macala LJ, Taki T, Weinfeld HM, Yu FS (1988) J Neurochem 50:1825 1829

13. Yu RK (1994) Prog Brain Res 101:31-44

14. Hakomori S (2000) Glycoconj J 17:627-647

15. Ledeen RW, Wu G (2002) Neurochem Res 27:637-647

16. Ziche M, Alessandri G, Gullino PM (1989) Lab Invest 61:629 634

17. Alessandri G, Kaju KS, Gullino PM (1986) Inv Metast 6:145-165 
18. Alssandri G, lle Cristan G, Ziche M, Cappa APM, Gullino PM (1992) J Cell Physiol 15l:23-28

19. Tai T, Kawashima I, Ozawa H, Kotani M, Ogura K (1997) Pure Appl Chem 69:1903-1910

20. Kracun I, Rosner H, Dmovsek V, Heffer-Lauc M, Cosovic C Lauc G (1991) Int J Dev Biol 35:289-295

21. Schnaar RL, Needham LK (1994) Methods Enzymol 230:371-389

22. Zamfir A, Vukelic Ž, Bindila L, Almeida R, Sterling A, Allen M, Peter-Katalinic J (2004) J Am Soc Mass Spectrom 15:1649-1657

23. Vukelic $\breve{Z}$, Bognar SK, Froesch M, Bîndilă L, Radić B, Allen M, Peter Katalinić J, Zamfir AD (2007) Glycobiology 17:504-5 I5

24. Vukelić Z, Zamfir AD, Bindila L, Froesch M, Peter-Katalinić J, Usuki S, Yu RK (2005) J Am Soc Mass Spectrom 16:571-580

25. Zamfir AD, Lion N, Vukelic Z, Bindila L, Rossier J, Girault HH, Peter-Katalinic J (2005) Lab Chip 5:298-307

26. Almeida $R$, Mosoarca $C$, Chirita $M$, Udrescu V, Dinca $N$, Vukelić Z, Allen M, Zamfir AD (2008) Anal Biochem 378:43-52

27. Serb A, Schiopu C, Flangea C, Vukelić Z, Sisu E, Dinca N, Zagrean L, Zamfir AD (2009) Eur J Mass Spectrom 15:541-553

28. Zamfir AD, Vukelic Ž, Schneider A, Sisu E, Dinca N, Ingendoh A (2007) J Biomol Tech 18:188 193
29. Serb A, Schiopu C, Flangea C, Sisu E, Zamfir AD (2009) $J$ Mass Spectrom in press

30. Svennerholm L, Fredman P (1980) Biochim Biophys Acta 617:97-109

31. Vuketić Z, Metelmann $W$, Müthing $J$, Kos M, Peter-Katalinić J (2001) Biol Chem 382:259-274

32. Domon B, Costello CE (1988) Glycoconjugate J 5:397-409

33. Costello CE, Juhasz P, Perreault H (1994) Progr Brain Res 101:45 61

34. IUPAC-IUB Joint Commission on Biochemical Nomenclature (1998) Eur J Biochem 257:293-298

35. Saito M, Sugiyama K (2000) Biochim Biophys Acta 1474:88-92

36. Visnapuu T, Zamfir AD, Mosoarca $C$, Stanescu MD, Alamäe $T$ (2009) Rapid Commun Mass Spectrom 23:1337-1346

37. Rouwendal GJ, Wuhrer M, Florack DE, Koeleman CA, Deelder AM, Bakker H, Stoopen GM, van Die I, Helsper JP, Hokke CH, Bosch D (2007) Glycobiology 17:334-344

38. Catalina MI, Koeleman CA, Deelder AM, Wuhrer M (2007) Rapid Commun Mass Spectrom 21:1053-1061

39. Wuhrer M, Koeleman CA, Deelder AM (2009) Anal Chem $81: 4422-4432$

40. Zeng G, Yu RK (2008) Curr Drug Targets 9:317-324 